\title{
Periodic Input Observer Design: Application for Imbalance Diagnosis
}

\author{
J. Chauvin, N. Petit, P. Rouchon
}

École des Mines de Paris

G. Corde

IFP

Copyright (C) 2006 Society of Automotive Engineers, Inc.

\begin{abstract}
Observation problems have been garnering increasing attention in recent years. They can be seen as the estimation of a periodic output dynamics driven by periodic inputs. At various level of modelling, automotive engine dynamics can be considered as a linear periodic system mechanically coordinated through the revolution of the crankshaft. In this paper, two practical examples are addressed. The first example is the inversion of sensor dynamics. A classical way of modelling such a sensor is a first order dynamics with periodic excitations which can be, depending on the application, the intake pressure, the intake temperature, the exhaust pressure, the Air Fuel Ratio, or the Mass Air Flow. The second example is the estimation of the engine speed next to the cylinder using as only sensor the easily available instantaneous crankshaft angle speed at the end of the connecting rod.
\end{abstract}

The contribution of this paper is the design of a real-time observer of the periodic excitation of a linear periodic systems by the estimation of the Fourier decomposition of the signal. The estimation of the coefficients of the Fourier basis decomposition of the input periodic excitation is a handy tool for engineering purposes. Indeed, the energy levels of the signal allow another interpretation of the signal and can lead to detect the balance of the engine. This high frequency ( $6^{\circ}$ crankshaft estimation) information can be used for imbalance diagnosis and torque balance control. Real application observation problems are exposed in their practical context and illustrated by experimental results on a 4 cylinder $\mathrm{HCCl}$ engine.

\section{INTRODUCTION}

Performance and environmental requirements impose advanced control strategies for automotive applications. Ide- ally, pressures, temperatures, and flows would be measured at numerous places in the engine, allowing accurate control. Unfortunately, their cost and reliability can prevent these sensors from reaching commercial products (e.g. in-cylinder pressure sensors). As a result, observer design has been garnering increasing attention in recent years. While each of the sensing technology poses its own challenges, several common threads can be found. In particular, many of these observation problems can be seen as the estimation of periodic dynamics driven by periodic inputs. At various level of modelling, automotive engine dynamics can be considered as a linear periodic system mechanically coordinated through the revolution of the crankshaft. A prime example is the inversion of sensor dynamics (Zone 1 on Figure 1) (see [1] and [2] for more details). A classical model of such a sensor is a first order dynamics with periodic excitation which can be, depending on the application, the intake pressure, the intake temperature, the exhaust pressure, the Air Fuel Ratio, or the Mass Air Flow.

A second example is the inversion of the transmission dynamics using as only sensor the instantaneous crankshaft angle speed (Zone 2 on Figure 1) which can lead to the estimation of the combustion torque (see [3, 4], [5], [6, 7, 8] for more details).

Imbalance is mostly produced by the injection system. Due to the high pressure in the common rail injection system, it is very difficult to estimate the fuel mass injected in the cylinder accurately. Moreover, aging of this system can create lag in the injection which leads to lag in the injection timing. Both problems imply the imbalance of the cylinder, i.e. with the same injection setpoint (injected masses and timing), the cylinder do not produce the same torque. A diagnosis based on commercial engine sensors is a great tool to solve this problem. 


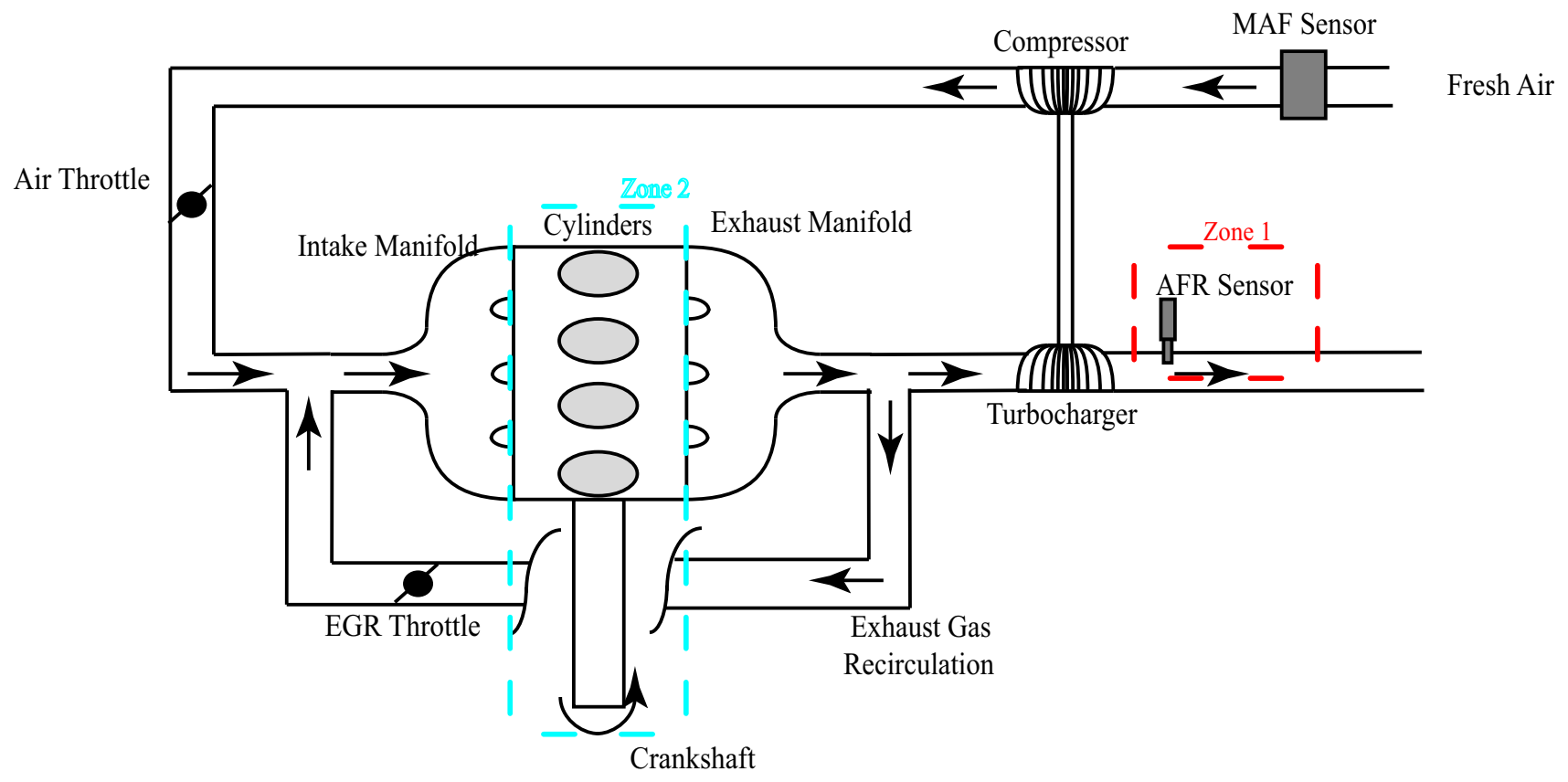

Figure 1: Engine Scheme.

In this paper, we cast such problem into the general framework of time-periodic linear systems driven by timeperiodic inputs $\dot{x}=A x+A_{0} w(t), y=C x$ where $x(t) \in \mathbb{R}^{n}$ and $w$ is a $T_{0}$-periodic signal containing a finite number of harmonics. The contribution of this paper is the design of real-time observers of the periodic excitation by estimation of its Fourier basis decomposition. By realtime observers, we mean that the computational power required by such asymptotic observers is quite low and thus fits with the real-time computers available on automotive engines. Among several alternatives, such observers may take the form of a filter complemented with adaptation laws for the decomposition coefficients which are weighted by periodic functions. Testbench results are presented proving the relevance of this kind of observer for torque balancing purpose.

The paper is organized as follows. In Section 2, we detail the problem statement and the observer structure. The first example of the AFR sensor dynamics inversion is reported is Section 3 along with testbench results. In Section 4 , an observer for the transmission rod dynamics inversion is explained. Testbench results present the use of this observer for torque balancing issues. Conclusion and future work are reported in Section 5.

\section{OBSERVATION PROBLEM STATEMENT}

Consider the periodic system driven by an unknown periodic input signal $w(t)$

$$
\left\{\begin{array}{l}
\dot{x}=A x+A_{0} w(t) \\
y=C x
\end{array}\right.
$$

where $x(t) \in \mathbb{R}^{n}$ is the state and $A, A_{0}, C$ are matrices in $\mathcal{M}_{n, n}(\mathbb{R}), \mathcal{M}_{n, m}(\mathbb{R})$ and $\mathcal{M}_{p, n}(\mathbb{R})$ respectively. The goal of our study is the estimation of the $T_{0}$-periodic continuous input signal $w(t) \in \mathbb{R}^{m}$, with $m=\operatorname{dim}(w) \leq p=\operatorname{dim}(y) \leq$ $n=\operatorname{dim}(x)$, through its Fourier decomposition over the $h$ harmonics

$$
w(t) \triangleq \sum_{k \in \mathcal{I}_{h}} c_{k} e^{\imath k \omega_{0} t} \omega_{0} \triangleq \frac{2 \pi}{T_{0}}
$$

where $\mathcal{I}_{h}=\{-h, \ldots, h\}$ is the set of $h$ modes. We assume that the following hypothesis hold

Hypothesis 1 (Injectivity of $A_{0}$ and $\left.C^{\dagger}\right) \operatorname{Ker}\left(A_{0}\right)=\{0\}$ and $\operatorname{Ker}\left(C^{\dagger}\right)=\{0\}$.

We note ${ }^{\dagger}$ the Hermitian transpose that reduces to standard transpose for matrices with real entries.

Hypothesis 2 (Observability) The only solution $t \mapsto$ $\left(x(t), c_{k}(t)\right)$ of

$$
\left\{\begin{aligned}
\dot{x} & =A x+A_{0}\left(\sum_{k \in \mathcal{I}_{h}} c_{k} e^{\imath k \omega_{0} t}\right) \\
\dot{c}_{k} & =0, \quad \forall k \in \mathcal{I}_{h}^{+}
\end{aligned}\right.
$$

for which the output $y(t)=C x(t)$ is identically zero on $\left[0, T_{0}\right]$, is the zero solution.

REFERENCE MODEL The reference system writes

$$
\left\{\begin{aligned}
\dot{x} & =A x+A_{0}\left(\sum_{k \in \mathcal{I}_{h}} c_{k} e^{\imath k \omega_{0} t}\right) \\
\dot{c}_{k} & =0, \quad \forall k \in \mathcal{I}_{h}, k \geq 0 \\
y & =C x
\end{aligned}\right.
$$

Generically we denote by $\mathcal{F}_{l}(x)$ the $l^{\text {th }}$ coefficient of the Fourier decomposition of a periodic signal $x(t)$, i.e. $\mathcal{F}_{l}(x)=\frac{1}{T_{0}} \int_{0}^{T_{0}} x(s) e^{-\imath l \omega_{0} s} d s$. When $x$ is real, the complex-conjugate of $\mathcal{F}_{l}(x)$ satisfies $\mathcal{F}_{l}(x)^{\dagger}=\mathcal{F}_{-l}(x)$. This explain the fact we consider only $k \in \mathcal{I}_{h}$ positive: $c_{k} \in \mathbb{C}$ when $k>0$ and $c_{k} \in \mathbb{R}$ when $k=0$. 
OBSERVER STRUCTURE Corresponding to statespace model (3), we define a time-varying Luenberger type observer

$$
\left\{\begin{array}{c}
\dot{\hat{x}}=A \hat{x}+A_{0}\left(\sum_{k \in \mathcal{I}_{h}} \hat{c}_{k} e^{\imath k \omega_{0} t}\right)-L(C \hat{x}-y) \\
\dot{\hat{c}}_{k}=-e^{-\imath k \omega_{0} t} L_{k}(C \hat{x}-y), \quad \forall k \in \mathcal{I}_{h}, k \geq 0
\end{array}\right.
$$

$L$ and $\left\{L_{k}\right\}_{k \in \mathcal{I}_{h}}$ are defined later through the convergence analysis. The error-state is $\tilde{x}=x-\hat{x}$ and $\tilde{c}_{k}=$ $c_{k}-\hat{c_{k}}$, and the error dynamics is

$$
\left\{\begin{array}{c}
\dot{\tilde{x}}=(A-L C) \tilde{x}+A_{0}\left(\sum_{k \in \mathcal{I}_{h}} \tilde{c}_{k} e^{\imath k \omega_{0} t}\right) \\
\dot{\tilde{c}}_{k}=-e^{-\imath k \omega_{0} t} L_{k} C \tilde{x}, \quad \forall k \in \mathcal{I}_{h}, k \geq 0
\end{array}\right.
$$

\section{OBSERVER DEFINITION}

Design of $L$ and $\left\{L_{k}\right\}_{k \in \mathcal{I}_{h}}$ with full measurement This subsection deals with the full state measurement case $p=n$. In this case, we use

$$
L=(A-\tilde{L}) C^{-1}(t)
$$

where $\tilde{L}$ be an asymptotic stable matrix in $\mathcal{M}_{n n}(\mathbb{R})$ and for all $k \in \mathcal{I}_{h}$, we choose the gains $L_{k}$ such that

$$
L_{k} \triangleq \alpha_{k}^{2} A_{0}^{\dagger} P C^{-1}
$$

where $P$ the symmetric definite solution of the Lyapunov equation

$$
P \tilde{L}+\tilde{L}^{\dagger} P=-I_{n, n}
$$

and $\left\{\alpha_{k}\right\}_{k \in \mathcal{I}_{h}} \in(\mathbb{R} \backslash\{0\})^{h}$. Convergence with this gains is proved in [9].

Design of $L$ and $\left\{L_{k}\right\}_{k \in \mathcal{I}_{h}}$ with partial measurement In this part, we consider that $p<n$. $L$ is chosen such that $A-L C$ is asymptotically stable. Assuming that for all $k \in \mathcal{I}_{h}$,

$$
P_{k} \triangleq C\left(\imath k \omega_{0}-P\right)^{-1} A_{0}
$$

is full rank, we choose the gains $L_{k}(t)$

$$
\left.\beta \neq 0 \text { and } L_{k}(t) \triangleq-\epsilon \beta^{2}\left(\left(\imath k \omega_{0}-(A-L C)\right)\right)^{-1} A_{0}\right)^{\dagger} C^{\dagger}
$$

For $\epsilon>0$ and small enough, convergence is proved [9].

\section{AIR FUEL RATIO SENSOR INVERSION}

The first example is the inversion of the Air Fuel Ratio (AFR) sensor. Its measurement is a very good representation of the combustion, especially in the case of cylinders imbalance on the engine (which are very frequent in practice). The individual cylinder exhaust flows are mixed in the exhaust manifold and passes through the turbocharger. This leads to a nonlinear dynamics as explained in [10] or [11] for example. The sensor has a lowpass dynamics.
SENSOR DYNAMICS In order to estimate the individual cylinder AFR, we need to estimate the AFR downstream the turbine and "invert" the sensor dynamics. The sensor can be considered as a cascade of two first order systems with time constant $\tau_{1}=25 \mathrm{~ms}$ and $\tau_{2}=20 \mathrm{~ms}$. $w$ is the input signal driving a cascade of two first order dynamics. The sensor is used to reconstruct in two steps $w$. First the intermediate variable $x_{1}$ is reconstructed under the form of a periodic signal. Second, this estimation feeds another observer. The structure is explained in Figure 2. For a first

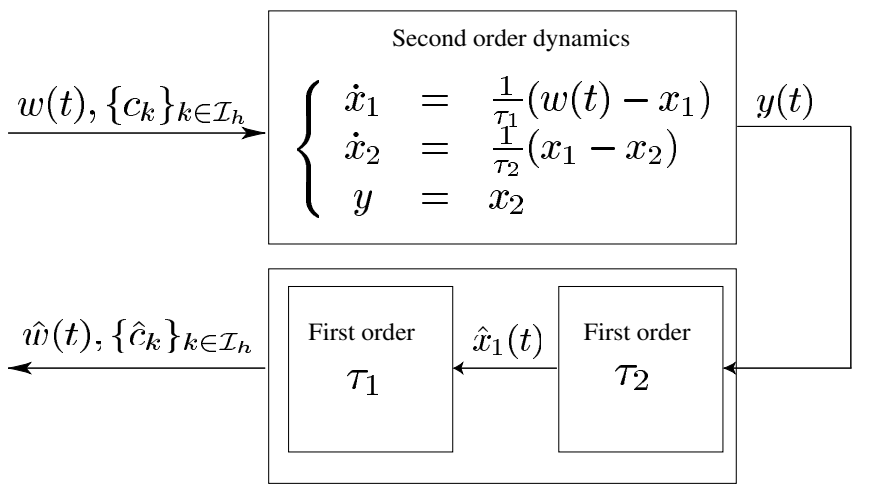

Figure 2: Top: $w$ is the input signal driving a cascade of two first order dynamics. Bottom: the sensor is used to reconstruct in two steps $w$. First the intermediate variable $x_{1}$ is reconstructed under the form of a periodic signal. Second, this estimation feeds another observer.

order system with a time constant $\tau$, the system is similar to system (3) with $A=-\frac{1}{\tau}, A_{0}=\frac{1}{\tau}, C=1$, and $\mathcal{I}_{h} \triangleq$ $\{-3, \ldots, 3\}$. The observer is designed according to (4) with the gains defined by Equations (6) and (7). We use $\tilde{L}=$ $-\frac{4}{\tau}$ and $\alpha_{k}^{2}=\frac{4}{k^{2}+1}$.

AFR OSCILLATIONS To introduce imbalance on the experimental testbed, we apply an injection duration timing trajectory. It produces offsets in injected masses leading to AFR disturbances. More precisely, the injection steps have an effect on the average level of the measured AFR and introduce oscillations as represented in Figure 3. These oscillations are the direct consequences of the individual AFR imbalance. During cylinder 1 exhaust phase, the AFR increases in the manifold, and then decreases while the other cylinders exhaust phases occur. The magnitude of the oscillations is related to the amount of the AFR difference between the cylinders and the gas mass in the manifold (and thus to its volume). The oscillation is then propagated to the turbine, and to the UEGO sensor, where it is filtered.

FROM OSCILLATIONS TO IMBALANCE DIAGNOSIS The implementation is achieved in discrete time with a sample angle of $6^{\circ} \mathrm{deg}$ crank angle. The observer described is evaluated on a 4 cylinder engine testbench, see [12]. At $t=4.5 \mathrm{~s}$, we introduce an imbalance on cylinder 1 ( $+25 \%$ of IMEP). This produces the expected oscillation of the AFR signal. The proposed technique is used 


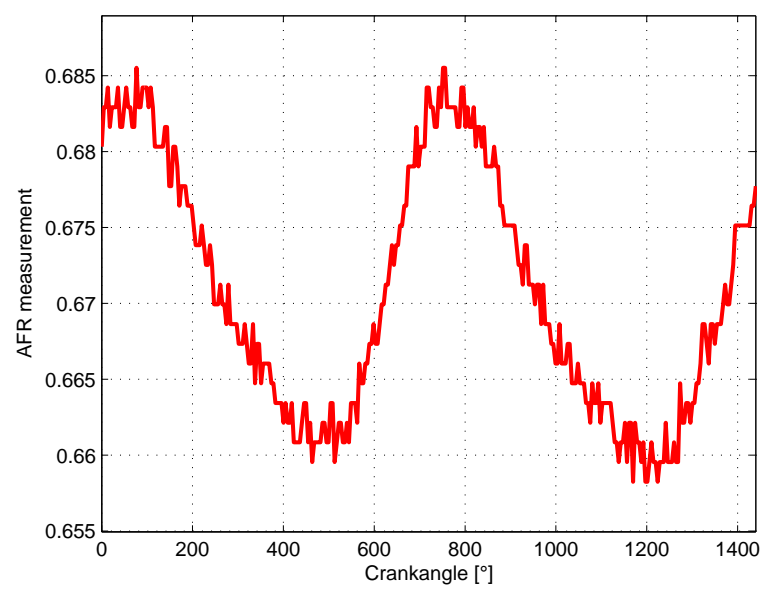

Figure 3: Testbench results. AFR oscillation after introduction of an imbalance on cylinder 1 ( $+25 \%$ of IMEP)

and provides the results reported in Figure 4.

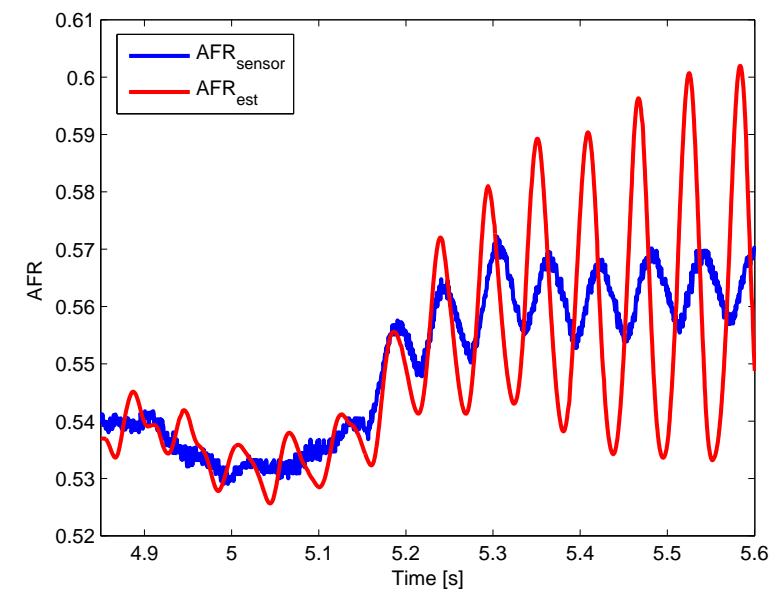

Figure 4: Testbench results. AFR reconstruction. Measured AFR by the sensor located downstream the turbine (blue), reconstructed AFR in exhaust manifold (red)

To check imbalance, a diagnosis function synchronized to the cylinders exhaust phases is applied. This function is the mean value of the AFR during the exhaust phase of each cylinder. Application of this diagnosis on both measured and estimated AFR is reported in Figures 5 and 7.

Moreover, the oscillation can be seen through the energy of the harmonics. For each harmonics, we define its energy by

$$
E_{c y l}(k)=\sqrt{\left\|c_{k}\right\|^{2}+\left\|c_{-k}\right\|^{2}}
$$

The energy of each harmonics is reported in Figures 6 and 8 . Notice that, as expected, the first harmonic raises for the imbalance of one cylinder and that only the second harmonics raises when two opposite cylinders are balanced each other. Without the observer, it is impossible to tell which cylinder is receiving an extra amount of fuel. The observer gives a reliable information on the dy-
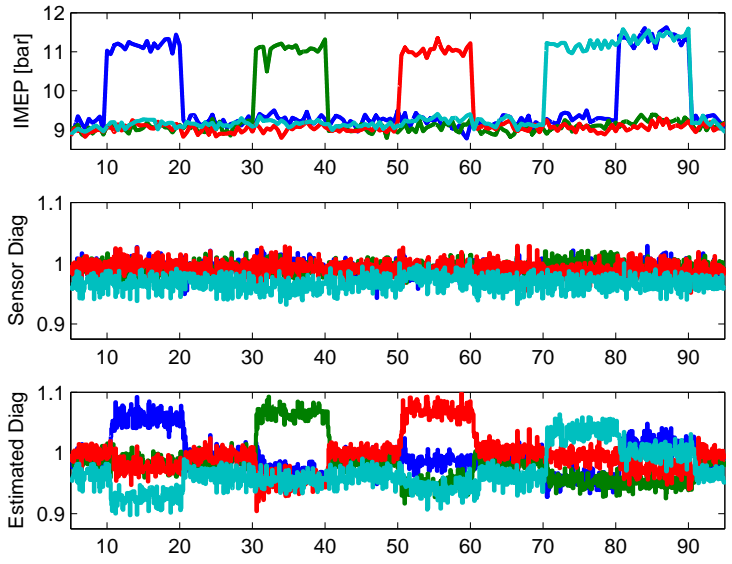

Figure 5: Testbench results. Diagnosis of cylinder imbalance. Top: Experimental IMEP produced by each cylinder. Middle: Diagnosis with AFR measurement. Bottom: Diagnosis with AFR estimation

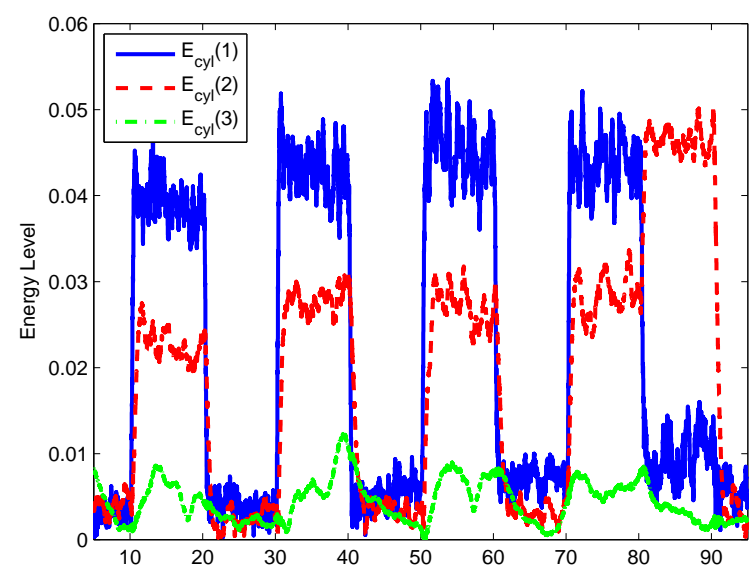

Figure 6: Testbench results. Energy level of each harmonics $E_{c y l}(k)=\sqrt{\left\|c_{k}\right\|^{2}+\left\|c_{-k}\right\|^{2}}$

namics of the signal, give an information on the imbalance (even if the information is not perfect, we are able to detect imbalance though the energy of the signal) and is a relevant tool towards individual cylinder Air Fuel Ratio.

TOWARDS TORQUE BALANCING As is, this information is not sufficient to elaborate an exact diagnosis, but combined to phasing with respect to the cylinders exhausts dynamics, it is a relevant input signal for an individual cylinder AFR to have a close estimation (as in [10] for example). This information could be used to balance the cylinder by applying a correction on the cylinder individual injection masses. Control of AFR imbalance between the cylinders can be addressed by controlling the individual injection quantities (which is the relevant control strategy for such imbalance) with a PI controller. Figure 9 presents the results of such a control strategy relying on the indi- 

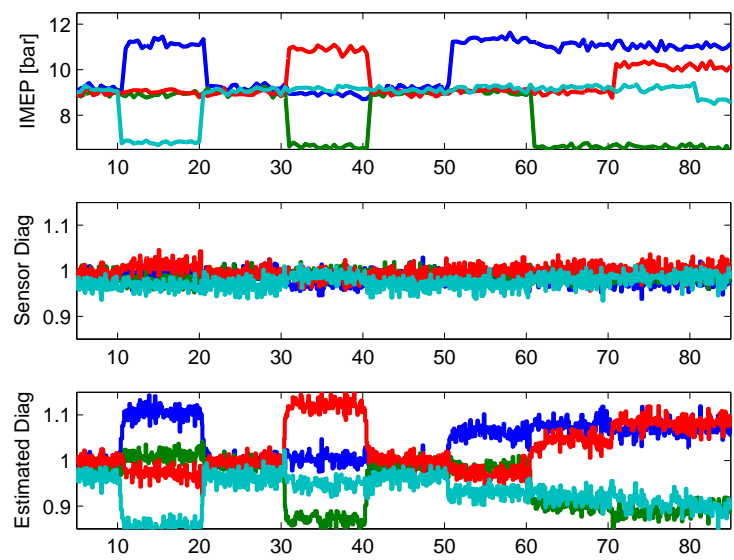

Figure 7: Testbench results. Diagnosis of cylinder imbalance. Top: Experimental IMEP produced by each cylinder. Middle: Diagnosis with AFR measurement. Bottom: Diagnosis with AFR estimation

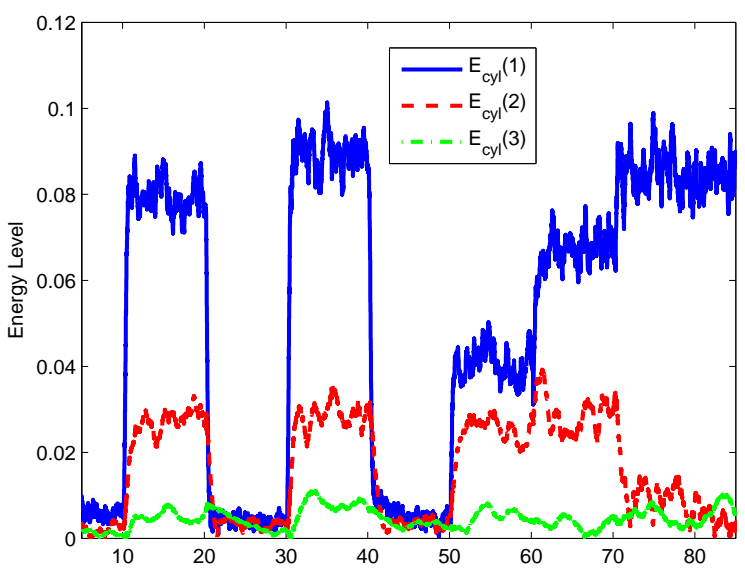

Figure 8: Testbench results. Energy level of each harmonics $E_{c y l}(k)=\sqrt{\left\|c_{k}\right\|^{2}+\left\|c_{-k}\right\|^{2}}$

vidual AFR estimation. On the testbench, we turn off the control at $7.5 \mathrm{~s}$ which leads to the natural imbalance of the engine. We introduce an injection offset on cylinder 2 at $19 s$ and finally turn the controller back on at $36 s$. We see that the control is efficient and that we are able to balance the torque produced by each cylinder.

\section{TRANSMISSION ROD DYNAMICS INVERSION}

For technical reasons, the instantaneous engine speed sensor is not located next to the cylinders but at the end of a transmission rod. When engine speed and torque increase, the excitation on the transmission rises in magnitude, yielding misleading information about the combustion. Modelling the transmission and inverting its dynamics is necessary for a good torque combustion estimation.

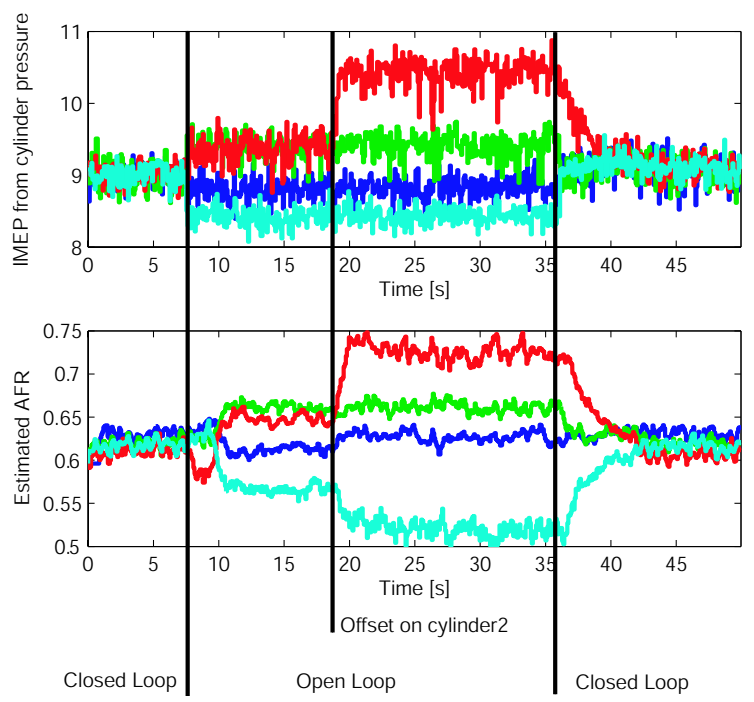

Figure 9: Torque balancing based on individual AFR estimation: test bench results (Engine Speed 1500rpm, IMEP $9 \mathrm{bar})$. We turn off the control at $7.5 \mathrm{~s}$, we introduce an injection offset on cylinder 2 at $19 \mathrm{~s}$ and finally turn the controller back on at 36s. Top: IMEP from cylinder pressure sensors. Bottom: Individual Estimated AFR with the nonlinear observer

MODEL DESCRIPTION Crankshaft dynamics modelling has been addressed previously in the literature (see [5] and [6] for example). In a first approach, the system can be modelled by a second order dynamics (classical for mechanical systems) as we can see in Figure 10. $x_{1}$ and $x_{2}$ refer to the engine speed at the end of the trans-

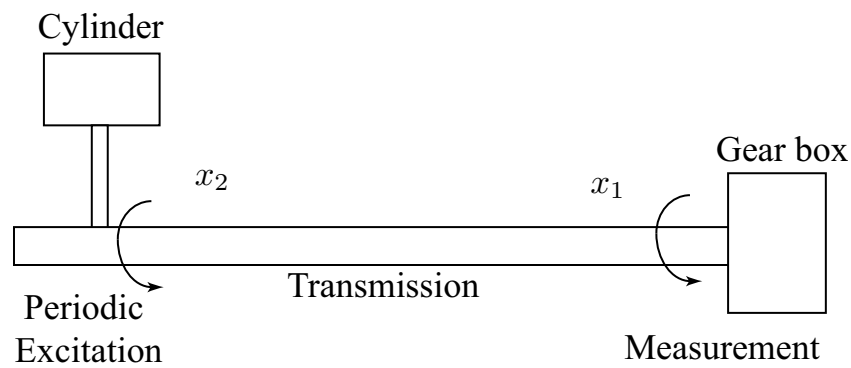

Figure 10: Transmission scheme.

mission and next to the cylinders respectively. The coupling dynamics writes

$$
\left\{\begin{array}{l}
\left(\frac{d^{2} x_{1}}{d \alpha^{2}}-\frac{d^{2} x_{2}}{d \alpha^{2}}\right)+2 \bar{\xi} \bar{\omega}\left(\frac{d x_{1}}{d \alpha}-\frac{d x_{2}}{d \alpha}\right)+\bar{\omega}^{2}\left(x_{1}-x_{2}\right)=0 \\
y=x_{1}
\end{array}\right.
$$

where $\bar{\xi}$ is a damping coefficient and $\bar{\omega}$ the natural frequency of the transmission. As the damping coefficient is much smaller than $1, .15$ in practice, we can not split the problem as previously. Let

$$
w_{0} \triangleq \frac{d^{2} x_{2}}{d \alpha^{2}}+2 \bar{\xi} \bar{\omega} \frac{d x_{2}}{d \alpha}+\bar{\omega}^{2} x_{2}
$$

as $x_{2}$ is mechanically periodic so does $w_{0}$. Some rewriting yields

$$
\left\{\begin{array}{l}
\frac{d^{2} x_{1}}{d \alpha^{2}}=-2 \bar{\xi} \bar{\omega} \frac{d x_{1}}{d \alpha}-\bar{\omega}^{2} x_{1}+w_{0} \\
y=x_{1}
\end{array}\right.
$$


OBSERVER DESIGN The state is $x=\left[\begin{array}{ll}x_{1} & \frac{d x_{1}}{d \alpha}\end{array}\right]^{\dagger}$. The state space model is of the form of system (3) with $A=\left[\begin{array}{cc}0 & 1 \\ -\bar{\omega}^{2} & -2 \bar{\xi} \bar{\omega}\end{array}\right], A_{0}=\left[\begin{array}{ll}0 & 1\end{array}\right]^{\dagger}$, and $C=$ $\left[\begin{array}{ll}1 & 0\end{array}\right]$. The observer is designed according to (4) with the gains defined by Equation (9) in subsection. We take $L=\left[\begin{array}{cc}2 \bar{\xi} \bar{\omega} & 2 \bar{\omega}^{2}\end{array}\right]^{\dagger}, \mathcal{I}_{h}=\{-2, \ldots, 2\}$ harmonics and $L_{k}(t)=-\epsilon \beta_{k}\left(\left(\imath k \omega_{0}-P\right)^{-1} A_{0}\right)^{\dagger} C^{T}$. We choose $\epsilon=0.1$ and $\beta^{2}=1$. The coefficients of the decomposition of $x_{2}$ are obtained from the coefficients of $w_{0}$ by matrix multiplication with

$$
\left[\begin{array}{cc}
\bar{\omega}^{2}-\left(k \omega_{0}\right)^{2} & k \omega_{0} 2 \bar{\xi} \bar{\omega} \\
-k \omega_{0} 2 \bar{\xi} \bar{\omega} & \bar{\omega}^{2}-\left(k \omega_{0}\right)^{2}
\end{array}\right]^{-1}
$$

TESTBENCH RESULTS The implementation is achieved in discrete time with a sample angle of $6^{\circ}$ deg crank angle. The observer described is evaluated on a 4 cylinder engine testbench, see [12]. The observer design yields the reconstruction of the engine speed next to the cylinder with the measurement of the engine speed and the end of the transmission. Experimental results are given in Figure 11. From these, we get an information on

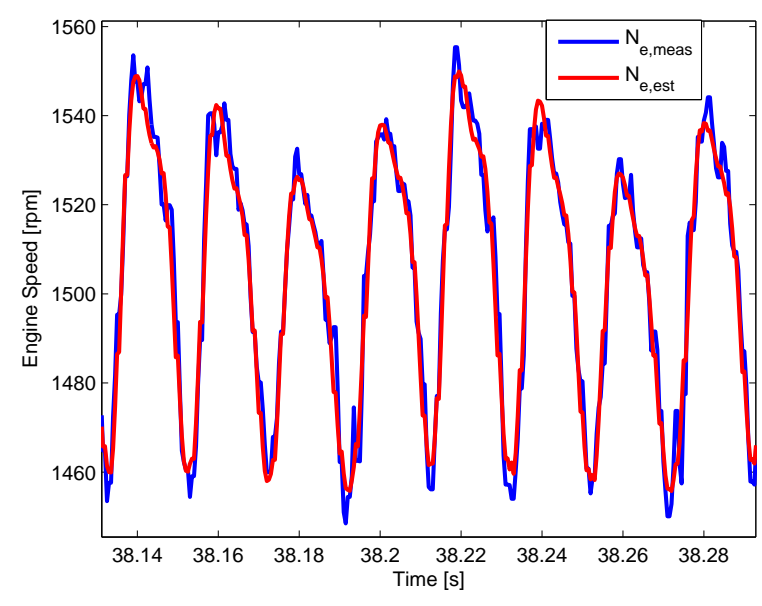

Figure 11: Testbench results. Engine speed reconstruction over 2 cycles at $1500 \mathrm{rpm}$ and 8 bar of IMEP: Blue : measured engine speed $N_{e, m e a s}$, reconstructed engine speed red : $N_{e, e s t}$

the work produced by each cylinder. This work is linked to the variance of the signal that writes

$$
V_{i}=\sum_{k \in \mathcal{I}_{h} \backslash\{0\}}\left\|c_{k, i}\right\|^{2}
$$

We can see the comparison between the IMEP produced by each cylinder (from the in-cylinder pressure measurement available on the testbench) and the variance of each cylinder in Figure 12.

\section{CONCLUSION AND FUTURE WORK}

In this paper, we expose an observation technique for a periodic linear system with an unknown periodic input
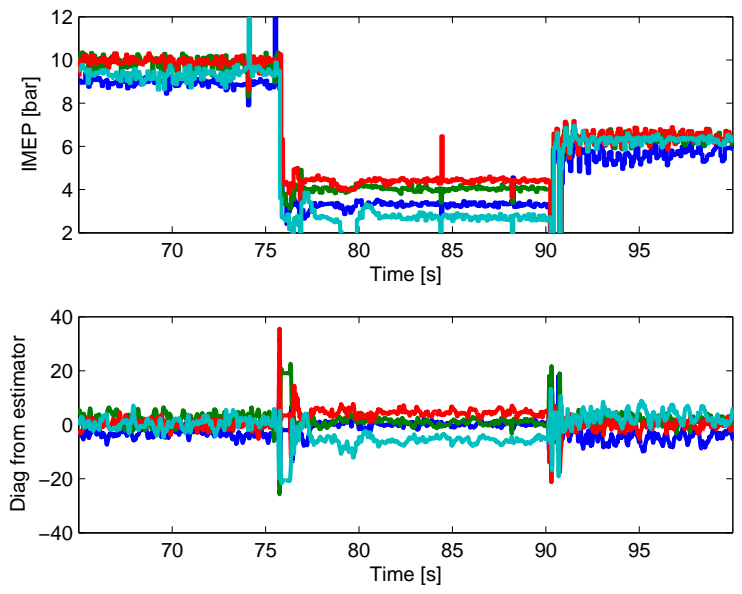

Figure 12: Testbench results. Diagnosis of torque imbalance. Top: IMEP from in-cylinder pressure measurement. Bottom: balance energy $\left(V_{i}-\frac{1}{n_{c y l}} \sum_{k} V_{k}\right.$, where $i$ refers to cylinder $i$ ) estimated with the observer.

along with several test cases from the automotive control systems area: torque estimation and sensors inversion. Our observer scheme estimates the state and the Fourier basis decomposition coefficients of the periodic excitation. The cases of full and partial state measurement are studied. In the first situation, our observer acts as a filter and proves efficient in practice: simplicity and low phase shift are among its reported properties. In the second case, the observer reconstructs unmeasured variables. The observation problems are exposed in their practical context and illustrated by experimental results. These studies suggest that periodic input estimation is a relevant technique for engine applications.

Moreover, the future work will focus on the use of the frequency information (the Fourier coefficients) for the engine control, i.e. correlate the Fourier coefficients with other parameters of the engine control. For example, we can try to estimate the EGR flow and the aspirated flow from the frequency information of the Manifold Air Flow and/or the intake pressure signal.

\section{References}

[1] J. Heywood. Internal Combustion Engine Fundamentals. McGraw-Hill, Inc, 1988.

[2] D. Hammerschmidt and $P$ Leteinturier. Automotive sensors and sensor interfaces. In Proc. of SAE Conference, number 2004-01-0210, 2004.

[3] L. Jianqiu, Y. Minggao, Z. Ming, and L. Xihao. Advanced torque estimation and control algorithm of diesel engines. In Proc. of SAE Conference, 2002.

[4] P. Gyan, S. Ginoux, J.-C. Champoussin, and Y. Guezennec. Crankangle based torque estimation: Mechanistic/stochastic. In Proc. of SAE Conference, 2000. 
[5] G. Rizzoni. Estimate of indicated torque from crankshaft speed fluctuations: A model for the dynamics of the IC engine. Proc. in the IEEE Transactions on Vehicular Technology, 38:169-179, 1989.

[6] G. Rizzoni and F. Connolly. Estimate of IC engine torque from measurment of crankshaft angular position. In Proc. of SAE Conference, 1993.

[7] J. Chauvin, G. Corde, P. Moulin, M. Castagné, N. Petit, and P. Rouchon. Real-time combustion torque estimation on a Diesel engine test bench using timevarying Kalman filtering. In Proc. of the the 43rd IEEE Conf. Decision and Control, 2004.

[8] J. Chauvin, G. Corde, P. Moulin, M. Castagné, N. Petit, and P. Rouchon. Real-time combustion torque estimation on a Diesel engine test bench using an adaptive Fourier basis decomposition. In Proc. of the the 43rd IEEE Conf. Decision and Control, 2004.

[9] J. Chauvin, P. Moulin, G. Corde, N. Petit, and P. Rouchon. High frequency reconstruction of automotive engine signals using periodic formulation. Automatica (Submitted), 2005.

[10] J. Chauvin, P. Moulin, G. Corde, N. Petit, and P. Rouchon. Real-time nonlinear individual cylinder Air Fuel Ratio observer on a Diesel engine test bench. In Proc. of the IFAC World Congress, 2005.

[11] C. Carnevale and M. Hadji. Cylinder to cylinder AFR control with an asymmetrical exhaust manifold in a GDI system. In Proc. of SAE Conference, number 981064, 1998.

[12] A. Albrecht, J. Chauvin, S. Potteau, and G. Corde. Design of real-time torque balancing control for highly premixed combustion engine using a $1 \mathrm{~d}$ Diesel engine model. In Proc. of the IAV Conference "Engine process simulation and supercharging", 2005. 